\title{
Physicochemical Characterisation of Polysaccharides from the Seeds and Leaves of Miracle Fruit (Synsepalum dulcificum) and Their Antioxidant and $\alpha$-Glucosidase Inhibitory Activities In Vitro
}

\author{
Huajun Jian, ${ }^{1,2}$ Fang Qiao, ${ }^{1}$ Jie Chen, ${ }^{3}$ and Nongyue $\mathrm{He}^{2}$ \\ ${ }^{1}$ School of Applied Chemistry and Biological Technology, Postdoctoral Innovation Practice Base, Shenzhen Polytechnic, \\ Shenzhen, Guangdong 518055, China \\ ${ }^{2}$ State Key Laboratory of Bioelectronics, School of Biological Science and Medical Engineering, Southeast University, \\ Nanjing, Jiangsu 210096, China \\ ${ }^{3}$ State Key Laboratory of Food Science and Technology, School of Food Science and Technology, Jiangnan University, \\ Wuxi, Jiangsu 214122, China \\ Correspondence should be addressed to Fang Qiao; qiaofang@szpt.edu.cn
}

Received 21 November 2016; Revised 24 January 2017; Accepted 2 March 2017; Published 30 March 2017

Academic Editor: Feng Xu

Copyright (C) 2017 Huajun Jian et al. This is an open access article distributed under the Creative Commons Attribution License, which permits unrestricted use, distribution, and reproduction in any medium, provided the original work is properly cited.

Miracle fruit (Synsepalum dulcificum) has been well known and studied for its unique taste-modifying ability. In this study, the monosaccharide composition, molecular weight $(\mathrm{Mw})$, and in vitro bioactivities (antioxidant, $\alpha$-glucosidase inhibition) of polysaccharides from the seeds (MFP-S) and leaves (MFP-L) of miracle fruit were investigated. The results showed that MFP-S was a homogeneous polysaccharide (Mw $2804 \mathrm{Da})$ with glucose. MFP-L displayed three fractions $(92093,1496$, and $237 \mathrm{Da})$ consisting of rhamnose, arabinose, galactose, glucose, and xylose. Moreover, the antioxidant and $\alpha$-glucosidase inhibition of MFP-L were significantly greater than those of MFP-S. The $\alpha$-glucosidase inhibition of MFP-L was remarkably better than the positive control, acarbose (an antidiabetes drug). More specifically, the 50\% inhibitory concentration (IC50) values of $\alpha$-glucosidase activities for MFP-S, MFP-L, and acarbose were 33, 0.01, and $1 \mathrm{mg} \mathrm{mL}^{-1}$, separately. Therefore, MFP-L can be developed as a functional factor with both antioxidant and antidiabetes activities in food applications.

\section{Introduction}

The miracle fruit (Synsepalum dulcificum) is an ever green indigenous to tropical west Africa. The shrub yields ripe red berries called "miracle fruit" that exhibit an interesting and remarkable taste-modifying property of altering sour flavours to sweet [1]. The pioneering study on miracle fruit was proposed by Inglett et al. [2] whilst looking for a natural sweetener to replace saccharin and cyclamate. Kurihara and Beidler [3] first reported in Science that the active ingredient could be a special glycoprotein with a molecular weight of $44 \mathrm{kDa}$, and Brouwer et al. [4] named the glycoprotein "miraculin" in Nature. Miraculin was further extracted, and its genes have been transferred to Aspergillus oryzae, lettuce, and tomato [5-7] to promote the mass production of this unique glycoprotein. In addition to miracle fruit's well-known tastemodifying ability, the fruit could be used to improve food palatability for patients who are undergoing chemotherapy [8].

It is noteworthy that all parts of this plant have medicinal importance [9]. However, Swamy et al. [10] found that few studies had been done on the medical or clinical effects of miracle fruit aside from well-established studies on the sweetening effects [11]. Inglett and Chen [12] investigated the antioxidant properties of phenolics and flavonoids in the skin, pulp, and seeds of miracle fruit and suggested it as a good source of antioxidants for functional food applications. He et al. [13] studied the total phenolic content and DPPH radical 
scavenging activities of extracts from the skin, pulp, and seed of miracle fruit. Du et al. [14] researched the phenolic and flavonoid content and antioxidant activity of miracle fruit flesh and seed methanol extracts, which could be used as an antioxidant-rich fruit to promote human health. Cheng et al. [15] also verified that polyphenols in seed extracts of miracle fruit were powerful antioxidants. Wang et al. [16] studied the antioxidant and antityrosinase effects of constituents of the stems of miracle fruit, suggesting the potential applications in food supplementation and medical cosmetology. Chen et al. [17] reported the inhibitory effects of aqueous extracts of miracle fruit leaves on oxidative and mutation damage and attributed them partially to its active phenolic components. Shi et al. [18] found that a butanol extract of miracle fruit exhibited equal effects to allopurinol, which is a commonly prescribed medication for gout and hyperuricemia. Therefore, they suggested that the butanol extract of miracle fruit could be used as a novel antihyperuricemia agent or health food. Moreover, Kaki Bale et al. [19] noted in a "Conference in Natural Medicine" that miracle fruit has been extensively used as an antidiabetes herbal medicine to protect the health of the Terengganu people in Malaysia, but the mechanism of effect has not been clarified. Chen et al. [20] suggested that miracle fruit could markedly raise insulin sensitivity in fructose-rich chow-fed rats, indicating that miracle fruit may be served as an adjuvant therapy for diabetic patients with insulin resistance.

Based on these very limited reports on the functional properties of polyphenol-enriched extracts from different parts of the miracle fruit plant, it was unexpected that polysaccharides have not yet been studied. However, it has been well reviewed that polysaccharides (such as tea polysaccharides) exhibit various bioactivities, including antioxidant, antidiabetes, anticancer, and immunological activities. Animal and clinical studies have suggested that tea polysaccharides play an important role in overall human health $[21,22]$. Many studies have been done to search for safe and efficient antioxidants and $\alpha$-glucosidase inhibitors from natural materials. Their purposes were to explore bioactive additives for functional foods or therapeutic compounds to prevent the development of diabetes and slow its progression. Nonstarch polysaccharides are also a type of dietary fiber, which can improve glycaemia and insulin sensitivity, lower good pressure, and drum cholesterol levels of individuals.

Therefore, we attempted to isolate polysaccharides from the seeds and leaves of miracle fruit. Their physicochemical properties (monosaccharide composition, molecular weight) and bioactivities, including antioxidant and $\alpha$-glucosidase inhibitory activities in vitro, were investigated in this study.

\section{Materials and Methods}

2.1. Materials and Chemicals. The fresh seeds and leaves of miracle fruit were provided by the Shenzhen Miracle Fruit Co. Ltd. (Shenzhen, Guangdong, China). 2,2-Diphenyl-1picrylhydrazyl (DPPH), 2,2' -azino-bis (3-ethylbenzothiazoline-6-sulfonic acid) (ABTS), Ferrozine, $\alpha$-glucosidase, and dextran molecular weight standards were purchased from Sigma Chemical Co. (St. Louis, USA). p-Nitrophenyl
$\alpha$-D-glucopyranoside ( $p$ NPG) and acarbose were purchased from J\&K Chemical Ltd. (Shanghai, China). All other chemicals and solvents were purchased from Sinopharm Chemical Reagent Co. Ltd. (Shanghai, China) and were of analytical grade or purer.

\subsection{Extraction and Isolation of Miracle Fruit Polysaccharides.} Polysaccharides are generally prepared with hot aqueous extraction followed by ethanol precipitation. The isolation processes of miracle fruit polysaccharides (MFPs) are presented in Figure 1. Both fresh seeds and leaves of miracle fruit were freeze-dried for $48 \mathrm{~h}$ in a Christ Alpha 1-4 LSC lyophiliser (Marin Christ, Osterode, Germany), were pulverised, passed through a 40 -mesh sieve $(0.43 \mathrm{~mm})$, and were stored at $-18^{\circ} \mathrm{C}$ in vacuum packaging. Each $60 \mathrm{~g}$ of the dried powder of seeds and leaves from miracle fruit was first processed ultrasonically with 5 volumes of $80 \%$ ethanol two times, in order to extract polyphenols, flavonoids, and oil. The dried residues were extracted with hot ultrapure water at $100^{\circ} \mathrm{C}$ at a ratio of $1: 10(\mathrm{w} / \mathrm{v})$ for $5 \mathrm{~h}$. Because the seeds contain a large amount of starch, which causes gelatinisation, $0.1 \% \alpha$-amylase was used to liquefy and decrease the viscosity of the extraction suspension at $70^{\circ} \mathrm{C}$.

After extraction, the supernatant solutions were collected by centrifugation $\left(6000 \mathrm{~g} ; 10 \mathrm{~min} ; 25^{\circ} \mathrm{C}\right)$ and concentrated to about $100 \mathrm{~mL}$ at $50^{\circ} \mathrm{C}$ using a R-215 rotary evaporator (Buchi, Zurich, Switzerland). Sevag reagent (chloroform: nbutyl alcohol $=4: 1, \mathrm{v} / \mathrm{v}$ ) was added and shaken to remove the proteins in concentrated solutions at a ratio of $1: 5(\mathrm{v} / \mathrm{v})$ for three times. The concentrated liquid extracts were mixed with 4 volumes of anhydrous ethanol at $4^{\circ} \mathrm{C}$ overnight for precipitation of MFPs. The resultant precipitates were collected by centrifugation $\left(6000 \mathrm{~g} ; 15 \mathrm{~min} ; 4^{\circ} \mathrm{C}\right)$. Polysaccharide precipitates were redissolved in water and precipitated with anhydrous ethanol and acetone twice again to remove other active compounds, soluble monosaccharide, and reducing sugars. At last, the polysaccharide precipitates were volatilised solvents and lyophilised to obtain the prepurified MFP fractions-those from seeds (MFP-S) and those from leaves (MFP-L).

2.3. Analysis of MFPs Monosaccharide Composition. The polysaccharide content of MFPs was determined by the phenol-sulfuric acid method using glucose as a standard [23]. The monosaccharide composition of MFPs was estimated by high-performance liquid chromatography (HPLC) after acid hydrolysis according to the method described by Wang et al. [24]. Briefly, each MFP sample (10 mg) was hydrolysed with trifluoroacetic acid (TFA, $2 \mathrm{M}, 3 \mathrm{~mL}$ ) at $121^{\circ} \mathrm{C}$ for $2 \mathrm{~h}$ in a sealed tube. Residual TFA was removed with a QGC-12T nitrogen blowing instrument (Quandao Co. Ltd., Shanghai, China) at $50^{\circ} \mathrm{C}$. The hydrolysed samples were redissolved in ultrapure water and analysed with ICS 5000 ion chromatography (Dionex, Sunnyvale, CA) with a CarboPac PA20 analytic column $(150 \mathrm{~mm} \times 3 \mathrm{~mm}$ inner diameter $)$ and a pulsed amperometric detector. The mobile phase consisted of $250 \mathrm{mM} \mathrm{NaOH}(2 \%)$ and water $(98 \%)$ at a flow rate of $0.5 \mathrm{~mL} \mathrm{~min}^{-1}$. 


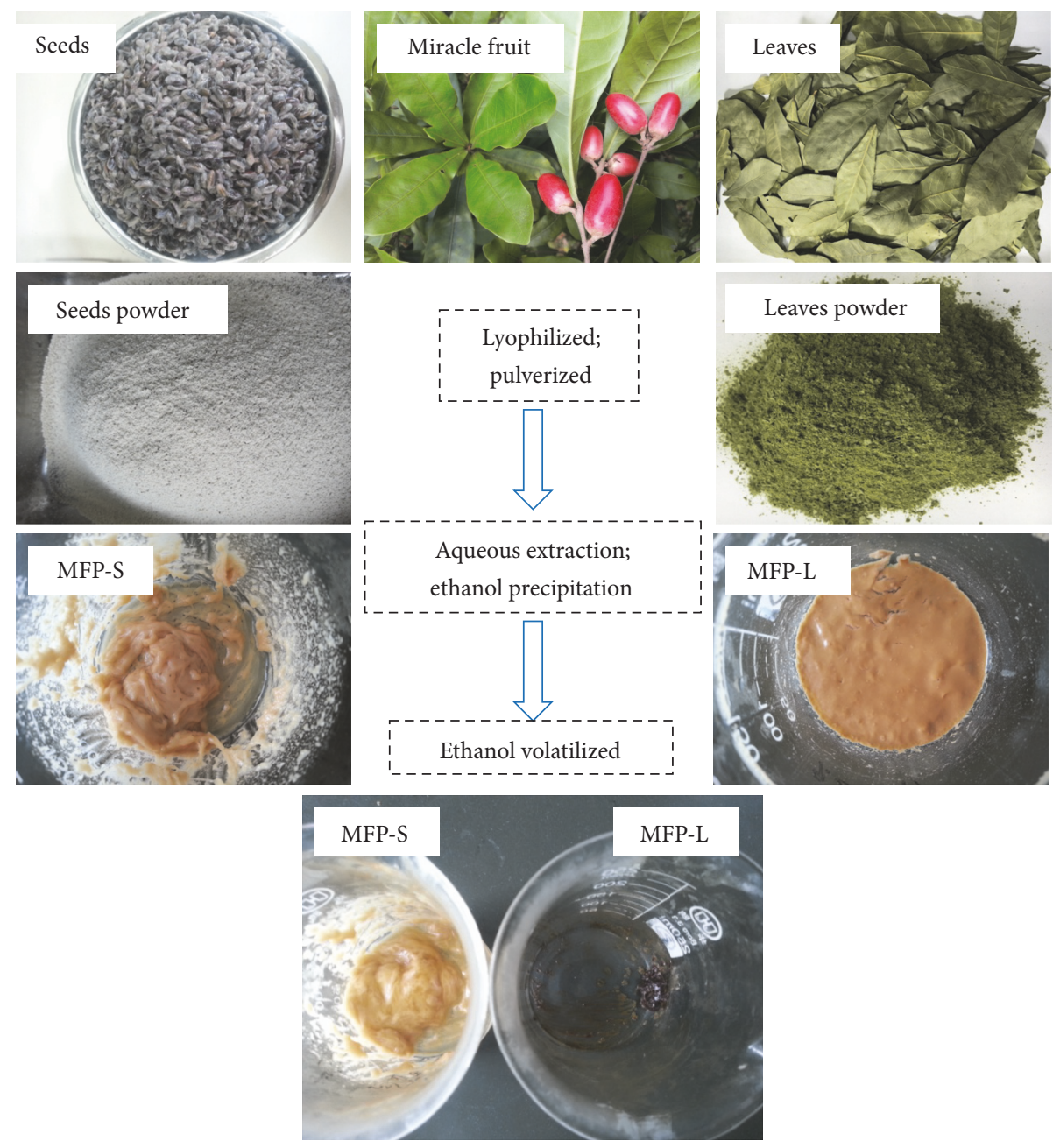

FIGURE 1: Preparation processes of MFP-S and MFP-L with aqueous extraction, followed by ethanol precipitation.

2.4. Determination of MFPs Molecular Weight. The molecular weight $(\mathrm{Mw})$ of MFPs was determined by high-performance gel-filtration chromatography (HPGFC), according to the method of Li et al. [25]. A Waters 1525 HPLC system was equipped with an Ultrahydrogel ${ }^{\mathrm{TM}}$ Linear $300 \mathrm{~mm} \times 7.8 \mathrm{~mm}$ inner diameter $\times 2$ (Waters Corp., Milford, MA), a Waters 2414 Refractive Index Detector (RI), and an Empower3 workstation. $\mathrm{NaNO}_{3}(0.1 \mathrm{M})$ was used as a mobile phase with a flow rate of $0.9 \mathrm{~mL} \mathrm{~min}^{-1}$. Dextran molecular weight standards ranging from 2.7 to $135 \mathrm{kDa}$ were used for calibration and calculation of the Mw of each peak.

2.5. Antioxidant Activities. In each of the following five different assays of antioxidant activities, the concentrations of MFP-S and MFP-L samples and the positive control of ascorbic acid (Vc) or ethylenediaminetetraacetic acid (EDTA) were set to be the same: MFP-S: $0.625,1.25,2.5,5,10,20,40$, and $80 \mathrm{mg} \mathrm{mL}^{-1}$; MFP-L: 0.00625, 0.0125, 0.025, 0.05, 0.1, 0.2, 0.4, and $0.8 \mathrm{mg} \mathrm{mL}^{-1}$; Vc/EDTA: 0.005, 0.01, 0.02, 0.04, 0.06, 0.08, 0.10 , and $0.12 \mathrm{mg} \mathrm{mL}^{-1}$.

2.5.1. DPPH Radical Scavenging Activity. The DPPH free radical scavenging activity of the MFPs was conducted according to a slightly modified method described by Li et al. [26]. In brief, $2 \mathrm{~mL}$ of the MFP samples at various concentrations was mixed with $4 \mathrm{~mL}$ of $0.2 \mathrm{mM}$ DPPH dissolved in $95 \%$ ethanol. The mixtures were shaken adequately and incubated for $20 \mathrm{~min}$ at $25^{\circ} \mathrm{C}$ in the dark. The absorbance was measured at $517 \mathrm{~nm}$ against a blank group. Ascorbic acid (Vc) was used as a positive control. The DPPH scavenging activity was calculated as follows:

$$
\begin{aligned}
& \text { DPPH scavenging activity }(\%) \\
& \quad=\left(1-\frac{A_{\text {sample }}-A_{\text {blank }}}{A_{\text {control }}}\right) \times 100,
\end{aligned}
$$

where $A_{\text {sample }}$ is defined as the absorbance of the sample (sample + DPPH methanol solution), $A_{\text {blank }}$ is the absorbance of the reagent blank (sample without DPPH methanol solution), and $A_{\text {control }}$ is the absorbance of the control (DPPH methanol solution without sample).

2.5.2. ABTS Radical Scavenging Activity. The ABTS radical scavenging activity of the MFPs was examined according to the method of Siddhuraju and Becker [27] with slight 
modification. The ABTS radical cation stock solution was prepared by mixing $88 \mu \mathrm{L}$ of $140 \mathrm{mM}$ potassium persulphate and $5 \mathrm{~mL}$ of $7 \mathrm{mM}$ ABTS and incubating for $24 \mathrm{~h}$ at $25^{\circ} \mathrm{C}$ in the dark. The ABTS radical stock solution was then diluted with ultrapure water to obtain an absorbance of $0.70 \pm 0.02$ at $734 \mathrm{~nm}$. MFP samples $(0.4 \mathrm{~mL})$ at various concentrations were excessively mixed with $6 \mathrm{~mL}$ of ABTS radical cation solution. The mixtures were left to stand for $1 \mathrm{~h}$ at $25^{\circ} \mathrm{C}$ in the dark, and the absorbance was tested at $734 \mathrm{~nm}$. Ascorbic acid was also used as a positive control. The ABTS scavenging activity was calculated as follows:

$$
\begin{aligned}
& \text { ABTS scavenging activity }(\%) \\
& \qquad=\left(1-\frac{A_{\text {sample }}-A_{\text {blank }}}{A_{\text {control }}}\right) \times 100,
\end{aligned}
$$

where $A_{\text {sample }}$ is defined as the absorbance of the sample, $A_{\text {blank }}$ is the absorbance of the reagent blank (sample without ABTS), and $A_{\text {control }}$ is the absorbance of the control (ABTS without sample).

2.5.3. Hydroxyl Radical Scavenging Activity. The hydroxyl radical scavenging activity of the MFPs was investigated according to a modified method proposed by Li et al. [26]. MFP samples $(3 \mathrm{~mL})$ at various concentrations were mixed with $0.5 \mathrm{~mL} \mathrm{FeSO}_{4}(6 \mathrm{mM})$ and $0.5 \mathrm{~mL} \mathrm{H}_{2} \mathrm{O}_{2}(6 \mathrm{mM})$, incubated for $10 \mathrm{~min}$, and added to $0.5 \mathrm{~mL}$ salicylic acid $(6 \mathrm{mM})$. The absorbance was measured at $510 \mathrm{~nm}$ after being left to stand for $30 \mathrm{~min}$ at $25^{\circ} \mathrm{C}$. Ascorbic acid was also used as a positive control. The hydroxyl radical scavenging activity was calculated as follows:

$$
\begin{aligned}
& \text { Hydroxyl radical scavenging } \operatorname{activity}(\%) \\
& =\left(1-\frac{A_{\text {sample }}-A_{\text {blank }}}{A_{\text {control }}}\right) \times 100
\end{aligned}
$$

where $A_{\text {sample }}$ is defined as the absorbance of the sample, $A_{\text {blank }}$ is the absorbance of the reagent blank (sample without reagents), and $A_{\text {control }}$ is the absorbance of the control (reagents without sample).

2.5.4. Ferrous Ion Chelating (FIC) Activity. The chelating effect of the MFPs on $\mathrm{Fe}^{2+}$ was monitored by measuring the formation of a ferrous iron-Ferrozine complex according to the method of Chew et al. [28] with minor modification. MFP samples $(4 \mathrm{~mL})$ at various concentrations were mixed with $150 \mu \mathrm{L} \mathrm{FeCl}_{2}(1 \mathrm{mM})$, incubated for $5 \mathrm{~min}$, and added to $0.6 \mathrm{~mL}$ Ferrozine $(1 \mathrm{mM})$. The absorbance was measured at $562 \mathrm{~nm}$ after being left to stand for $10 \mathrm{~min}$ at $25^{\circ} \mathrm{C}$. EDTA was used as the positive control. The ferrous ion chelating ability was calculated with the following equation:

Ferrous ion chelating activity (\%)

$$
=\left(1-\frac{A_{\text {sample }}-A_{\text {blank }}}{A_{\text {control }}}\right) \times 100 \text {, }
$$

where $A_{\text {sample }}$ is defined as the absorbance of the sample, $A_{\text {blank }}$ is the absorbance of the reagent blank (sample without reagents), and $A_{\text {control }}$ is the absorbance of the control (reagents without sample).
2.5.5. Ferric-Reducing Antioxidant Power. The ferric-reducing antioxidant power (FRAP) assay was performed following a modified method of Wang et al. [24]. MFP samples $(0.5 \mathrm{~mL})$ at various concentrations were mixed with $2 \mathrm{~mL}$ phosphatebuffered saline solution (PBS; 0.2 M, pH 6.6) and $1 \mathrm{~mL}$ potassium ferricyanide $(1 \%, \mathrm{w} / \mathrm{v})$, separately, incubated in a $50^{\circ} \mathrm{C}$ water bath for $20 \mathrm{~min}$ and cooled immediately. After $2.5 \mathrm{~mL}$ trichloroacetic acid $(10 \%, \mathrm{w} / \mathrm{v})$ was added, the reaction mixture was centrifuged $\left(5000 \mathrm{~g} ; 10 \mathrm{~min} ; 25^{\circ} \mathrm{C}\right)$ and $1 \mathrm{~mL}$ of the supernatant was mixed with $2 \mathrm{~mL}$ ultrapure water and $0.5 \mathrm{~mL}$ $\mathrm{FeCl}_{3}(0.1 \%$, w/v). The absorbance was measured at $700 \mathrm{~nm}$ after being left to stand for $10 \mathrm{~min}$ at $25^{\circ} \mathrm{C}$. Ascorbic acid was also used as a positive control. The higher absorbance indicated better reducing power.

2.6. $\alpha$-Glucosidase Inhibition Assay. The $\alpha$-glucosidase inhibitory activity of the MFPs was determined according to Xu et al. [29] with slight modification. A series of polysaccharide samples at various concentrations was prepared: 10, 20, 40, 80, 160, 320, and $640 \mathrm{mg} \mathrm{mL}^{-1}$ for MFP-S and 0.0025 , $0.005,0.01,0.02,0.04$, and $0.08 \mathrm{mg} \mathrm{mL}^{-1}$ for MFP-L. MFP samples $(0.6 \mathrm{~mL})$ were mixed with $0.4 \mathrm{~mL}$ PBS (0.1 M, pH 6.9) containing $\alpha$-glucosidase $\left(0.1 \mathrm{U} \mathrm{mL}^{-1}\right)$ and incubated in a $37^{\circ} \mathrm{C}$ water bath for $10 \mathrm{~min}$. Afterward, $170 \mu \mathrm{L}$ of $1 \mathrm{mM} p$ nitrophenyl $\alpha$-D-glucopyranoside ( $p N P G$ ) as a substrate in PBS (0.1 M, pH 6.9) was added to the mixture. The reaction solution was incubated at $37^{\circ} \mathrm{C}$ for $30 \mathrm{~min}$, and the reaction was stopped by adding $2 \mathrm{~mL}$ of $0.5 \mathrm{M} \mathrm{Na} \mathrm{CO}_{3}$. The $\alpha$ glucosidase inhibitory activity was determined by monitoring the absorbance of $p$-nitrophenol release from $p N P G$ at $405 \mathrm{~nm}$. Acarbose $\left(0.02,0.2,2,4,8\right.$, and $\left.16 \mathrm{mg} \mathrm{mL}^{-1}\right)$ was used as the positive control. $\alpha$-Glucosidase inhibitory activity was calculated with the following equation:

$$
\begin{aligned}
& \text { Inhibition activity (\%) } \\
& \qquad=\left(1-\frac{A_{\text {sample }}-A_{\text {blank }}}{A_{\text {control }}}\right) \times 100,
\end{aligned}
$$

where $A_{\text {sample }}$ is defined as the absorbance of the sample, $A_{\text {blank }}$ is the absorbance of the reagent blank (sample + PBS instead of other reagents), and $A_{\text {control }}$ is the absorbance of the control (PBS instead of sample).

2.7. Statistical Analysis. All experiments were performed at least twice each with triplicate sample analysis. The results are expressed as means \pm SD. The method of analysis was based on the general linear model. Significant differences (set at $P<$ 0.05 ) between means were distinguished by least significant difference procedures using Statistix software 9.0 (Analytical Software, Tallahassee, FL, USA).

\section{Results and Discussion}

3.1. Monosaccharide Compositions of MFP-S and MFP-L. The yield of the polysaccharides MFP-S and MFP-L was $15.5 \%$ and $1.0 \%$, respectively. An HPLC chromatogram of mixed standard monosaccharides is presented in Figure 2(a). From left to right peaks 1-10 were amino glucose, rhamnose, arabinose, galactosamine, galactose, glucose, xylose, mannose, 


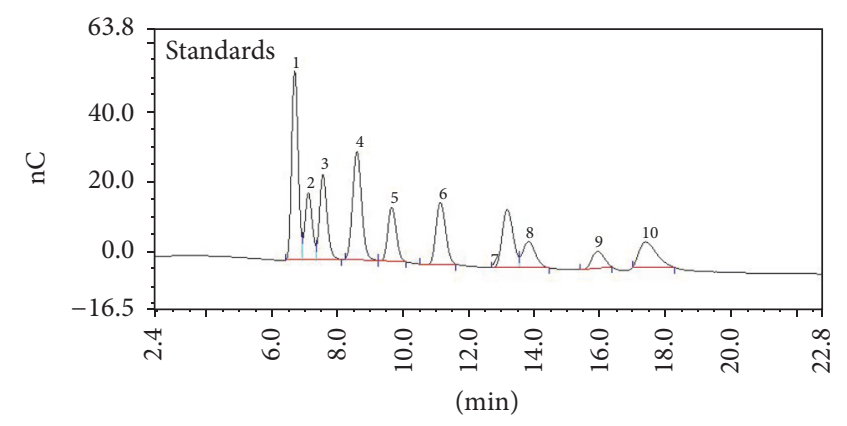

(a)

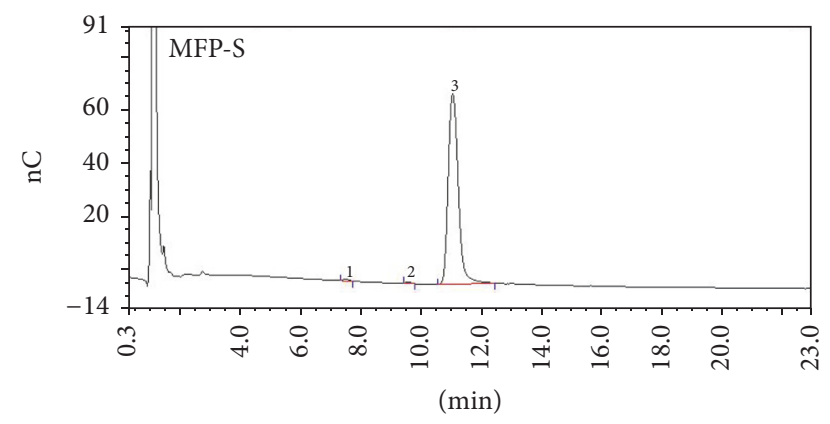

(b)

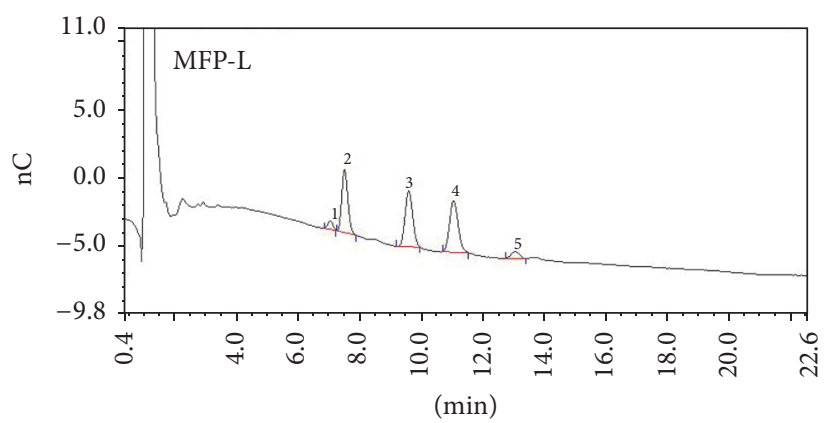

(c)

FIGURE 2: HPLC chromatograms of standard monosaccharides (a) and monosaccharide composition analysis of MFP-S (b) and MFP-L (c).

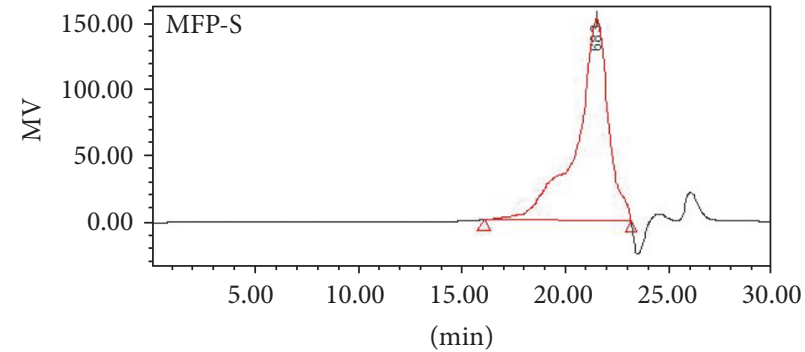

(a)

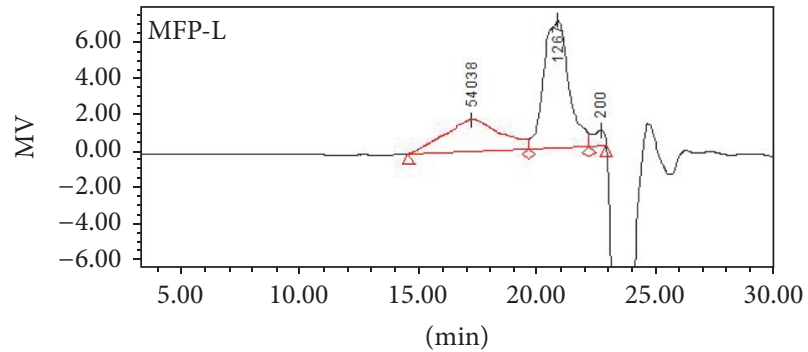

(b)

FIGURE 3: HPGFC profiles of MFP-S (a) and MFP-L (b) monitored with a refractive index detector.

fructose, and ribose, respectively. The results indicate that MFP-S and MFP-L were both heteropolysaccharides. MFP-S consisted of arabinose, galactose, and glucose in a ratio of $0.16: 0.09: 27.35$ (Figure 2(b)). In contrast, MFP-L was composed of five monosaccharides, rhamnose, arabinose, galactose, glucose, and xylose, with ratios of $0.12: 1.05: 1.17$ : 1.21: 0.15 (Figure 2(c)). Actually, the differences in MFP-S and MFP-L on their monosaccharides could be predicted during the isolation process. That is, MFP-S exhibited high viscosity like glucose syrup, whilst MFP-L had a low viscosity.

3.2. Molecular Weight Distributions of MFP-S and MFP-L. The homogeneity and molecular weight $(\mathrm{Mw})$ distributions of MFP-S and MFP-L were analysed by HPGFC. The average $\mathrm{Mw}$ of each elution peak was calculated according to the calibration curve derived from molecular weight standards. As shown in Figure 3(a), MFP-S presented a single, sharp, and symmetrical peak with an approximate $\mathrm{Mw}$ of $2804 \mathrm{Da}$ (Mp: $683 \mathrm{Da}$ ), implying that MFP-S was a homogeneous polysaccharide. This result is consistent with its almost single monosaccharide composition with glucose (Figure 2(b)). Li et al. [25] reported a similar HPGFC profile for polysaccharide. MFP-L consisted of three major fractions with $\mathrm{Mw}$ of 92093, 1496, and $237 \mathrm{Da}$ (Mp: 54038, 1267, and $200 \mathrm{Da}$ ) in proportion of $34 \%, 62 \%$, and $4 \%$, respectively (Figure $3(\mathrm{~b})$ ). The average Mw of three fractions in MFP-L was calculated to be $32249 \mathrm{Da}$.

3.3. Antioxidant Activities of MFP-S and MFP-L. The antioxidant activities of MFP-S and MFP-L with positive control were simultaneously estimated using five different methods, including DPPH, ABTS, hydroxyl radical scavenging activity, ferrous chelating ability, and ferric-reducing antioxidant power.

3.3.1. DPPH Radical Scavenging Activity. Because DPPH radicals are very stable but can be easily scavenged by antioxidants, they have been used to evaluate the free radical scavenging activity of natural compounds. As shown in Figure 4(a), all MFP-S, MFP-L, and positive control (Vc) scavenged 


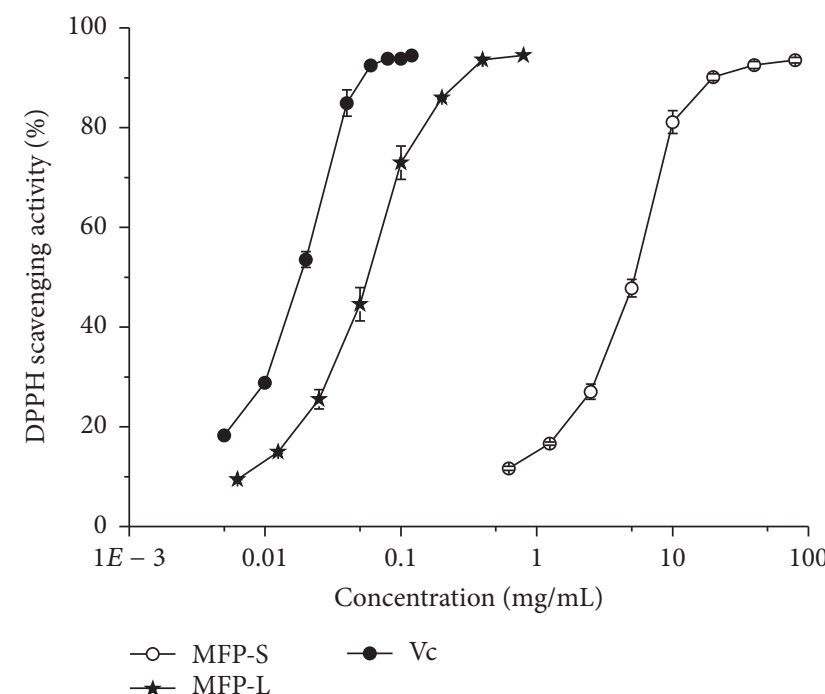

(a)

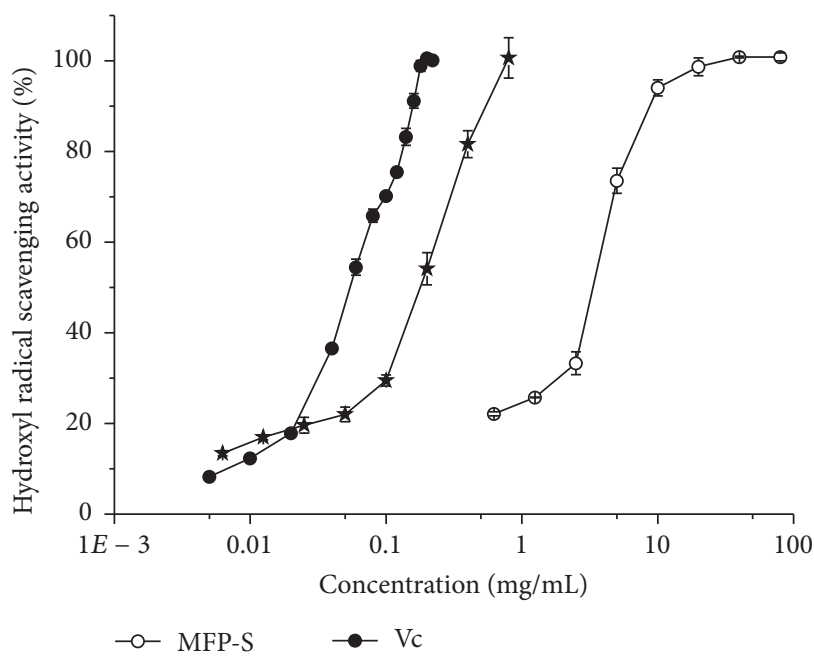

(c)

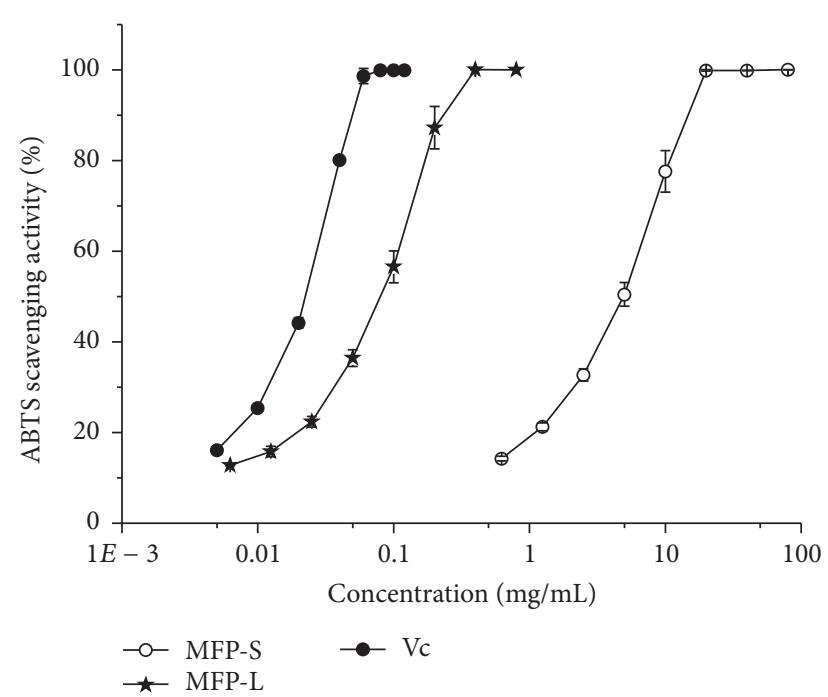

(b)

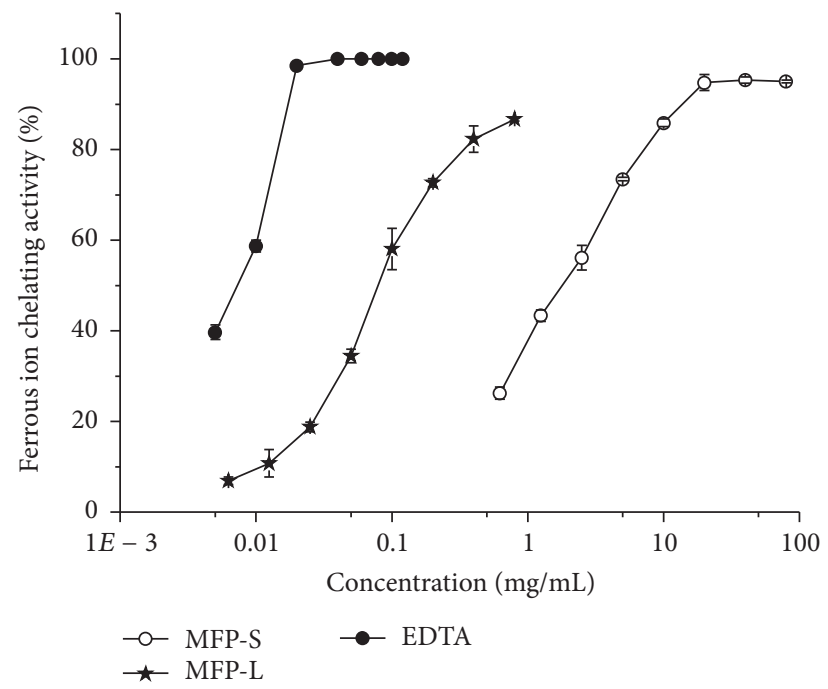

(d)

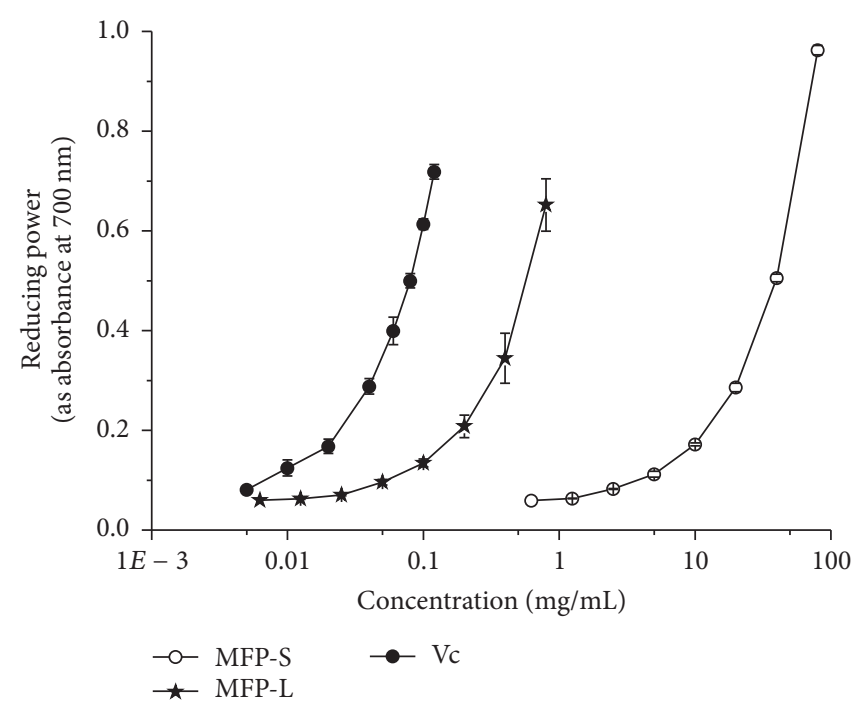

(e)

FIGURE 4: Antioxidant activities of MFP-S and MFP-L in vitro. DPPH (a); ABTS (b); hydroxyl radical scavenging activity (c); ferrous ion chelating activity (d); and ferric-reducing antioxidant power (e). 
$\mathrm{DPPH}$ radicals in a concentration-dependent manner at their own appropriate concentration ranges. Remarkably, MFP$\mathrm{L}$ presented much greater scavenging activity than MFPS. Calculations were done to identify concentrations that caused 50\% inhibition (IC50) of antioxidant activities. More specifically, the IC50 values of DPPH for MFP-S, MFP-L, and Vc were 5.3, 0.06, and $0.019 \mathrm{mg} \mathrm{mL}^{-1}$, separately. Therefore, MFP-L could possibly act as a strong DPPH radical scavenger in the food application.

3.3.2. ABTS Radical Scavenging Activity. The ABTS radical scavenging activities of MFP-S, MFP-L, and Vc were determined, and the results are shown in Figure 4(b). Similar to the results of DPPH (Figure 4(a)), the ABTS radical scavenging activities of MFP-S, MFP-L, and Vc were also increased in a concentration-dependent manner. MFP-L was found to be a more effective ABTS radical scavenger than MFP-S. For example, the IC50 values of ABTS for MFP-S, MFP-L, and Vc were $5.0,0.084$, and $0.023 \mathrm{mg} \mathrm{mL}^{-1}$, separately. It was implied that MFP-L could be used as a natural antioxidant to scavenge ABTS free radicals. This result also confirmed the positive correlation between ABTS and DPPH radical assays about antioxidant activity [30].

3.3.3. Hydroxyl Radical Scavenging Activity. Hydroxyl radicals are well known as some of the most reactive free radicals, which can induce severe damage to the organism [31]. Therefore, scavenging activities on the hydroxyl radical generated in a Fenton reaction of MFP-S, MFP-L, and Vc were investigated in this study. It could be seen that MFP-L still exhibited significantly better hydroxyl radical scavenging activity than MFP-S, as shown in Figure 4(c). In detail, the IC50 values of hydroxyl radical for MFP-S, MFP-L, and Vc were $3.5,0.18$, and $0.055 \mathrm{mg} \mathrm{mL}^{-1}$, separately.

3.3.4. Ferrous Ion Chelating (FIC) Activity. The metal chelating ability was recognised as a correlative activity to antioxidant. As presented in Figure 4(d), at each considered concentration range, the FIC activities of MFP-S, MFP-L, and EDTA increased as the polysaccharide concentration increased. MFP-L also displayed a distinct advantage over MFP-S on FIC activity, whilst the overall FIC activity was not as strong as that of EDTA. Specifically, the IC50 values of FIC activity for MFP-S, MFP-L, and EDTA were 1.9, 0.083, and $0.008 \mathrm{mg} \mathrm{mL}^{-1}$, separately.

3.3.5. Ferric-Reducing Antioxidant Power (FRAP). FRAP was expressed as absorbance (ABS) at $700 \mathrm{~nm}$, and a higher absorbance value indicated stronger reducing power. The results of FRAP assay are shown in Figure 4(e). As expected, Vc possessed the best FRAP, followed by MFP-L and MFPS. MFP-L still markedly exhibited much stronger activity for FRAP than MFP-S. The ABS of MFP-L at $0.00625 \mathrm{mg} \mathrm{mL}^{-1}$ was 0.060 , which was already comparable to the ABS of MFP-S at initial $0.625 \mathrm{mg} \mathrm{mL}^{-1}$. At the last point of MFP-L at $0.8 \mathrm{mg} \mathrm{mL}^{-1}$, the right ABS was 0.652 , whilst MFP-S was more than $40 \mathrm{mg} \mathrm{mL}^{-1}$, and $\mathrm{Vc}$ was less than $0.12 \mathrm{mg} \mathrm{mL}^{-1}$.

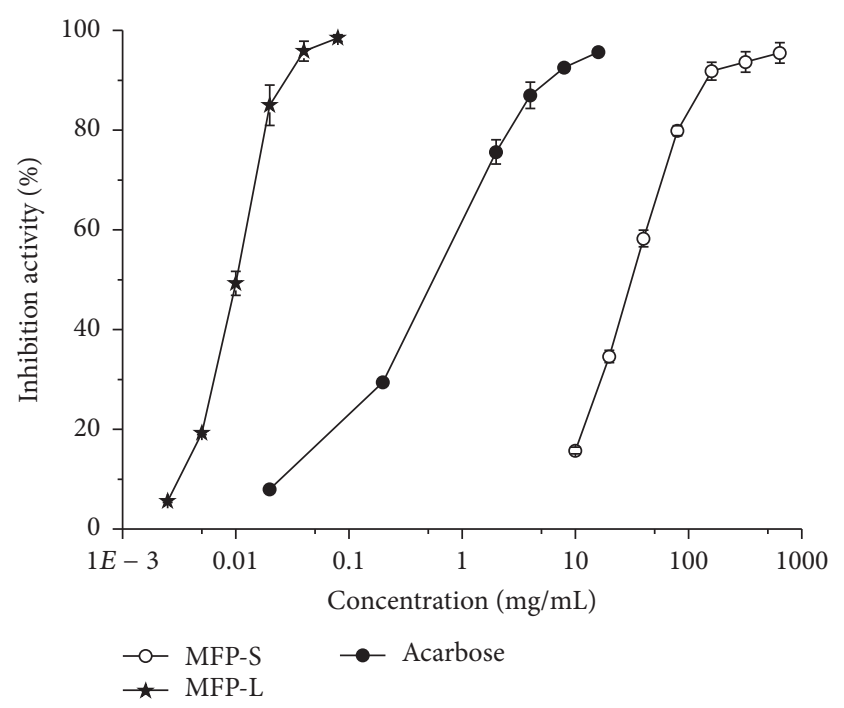

FIGURE 5: $\alpha$-Glucosidase inhibitory activities of MFP-S and MFP-L with acarbose used as positive control.

3.4. $\alpha$-Glucosidase Inhibitory Activity. Diabetes is characterised as a high concentration of blood sugar that can cause serious complications, such as damage to the eyes, kidneys, and cardiovascular and nervous systems. $\alpha$-Glucosidase is an important carbohydrate digestion enzyme that has been considered as a therapeutic approach to adjust postprandial hyperglycaemia [29]. Many studies have focused on seeking effective and safe $\alpha$-glucosidase inhibitors from natural products to develop functional foods to combat diabetes [32-36].

The dose-dependent effects on $\alpha$-glucosidase inhibitory activities of MFP-S, MFP-L and the positive control acarbose are displayed in Figure 5. It was amazing to note that MFP$\mathrm{L}$ presented remarkably greater $\alpha$-glucosidase inhibitory activity than MFP-S and greater even than acarbose. More specifically, the inhibitory ability of MFP-L rose from $5.6 \%$ to $98.5 \%$ when the concentration was increased from 0.0025 to $0.08 \mathrm{mg} \mathrm{mL}^{-1}$. More specifically, the IC50 values of $\alpha$ glucosidase activities for MFP-S, MFP-L, and acarbose were 33, 0.01 , and $1 \mathrm{mg} \mathrm{mL}^{-1}$, separately. This finding indicates that MFP-L could have a promising inhibitory effect on $\alpha$ glucosidase, even superior to that of the antidiabetes drug acarbose. Interestingly, it is coincident that the blood sugar values of some people with hyperglycaemia can be decreased to some extent by drinking tea made from the leaves of miracle fruit (data not shown).

Similarly, according to Xu et al. [29], the polysaccharide isolated from pu-erh tea stored for 5 years inhibits $\alpha$ glucosidase three times better than that from acarbose. Chen et al. [32] considered that the diversities in antioxidant and $\alpha$-glucosidase inhibitory activities amongst the three polysaccharides from green, oolong, and black tea appeared to be related to differences in their respective monosaccharide compositions and molecular weight distributions. They found that the high proportion of low molecular weight fractions in black tea polysaccharide contributed to its greater bioactivities of antioxidant and $\alpha$-glucosidase inhibition than those in green and oolong tea. According to the results of 
molecular weight distribution analysis of MFP-S and MFP-L (Figure 3), two fractions (Mw 1496 and 237 Da, resp.) of MFPL accounted for $66 \%$, whilst the single fraction of MFP-S had a higher average $\mathrm{Mw}$ of $2804 \mathrm{Da}$. It seems to be reasonable that low molecular weight fractions of MFP-L react easily with free radicals and other substrates to display wonderful bioactivity.

\section{Conclusions}

Overall, MFP-S and MFP-L have considerable differences in physicochemical properties (monosaccharide composition and molecular weight) and antioxidant and $\alpha$-glucosidase inhibitory activities in vitro. The results demonstrate that MFP-S was a homogeneous polysaccharide that consisted almost entirely of glucose. However, MFP-L was a heterogeneous polysaccharide with three elution fractions, which were made up of five monosaccharides: rhamnose, arabinose, galactose, glucose, and xylose. MFP-L exhibited much better antioxidant abilities than MFP-S, although they were still weaker than those of the positive control (Vc/EDTA). The most exciting feature was that MFP-L showed notable inhibition of $\alpha$-glucosidase, which was much better than that of the antidiabetes drug acarbose. It is suggested that MFP$\mathrm{L}$ can be explored as a novel antidiabetes additive with both antioxidant and hypoglycemic bioactivities. The relationship between the structure of purified fractions from MFP-L and their bioactivities should be considered in further studies.

\section{Conflicts of Interest}

The authors declare that they have no conflicts of interest regarding the publication of this paper.

\section{Acknowledgments}

This research work was financially supported by the authors' home institution funds.

\section{References}

[1] G. Igarashi, R. Higuchi, T. Yamazaki, N. Ito, I. Ashida, and Y. Miyaoka, "Differential sweetness of commercial sour liquids elicited by miracle fruit in healthy young adults," Food Science and Technology International, vol. 19, no. 3, pp. 243-249, 2013.

[2] G. E. Inglett, B. Dowling, J. J. Albrecht, and F. A. Hoglan, "Taste-modifying properties of miracle fruit (Synsepalum dulcificum)," Journal of Agricultural and Food Chemistry, vol. 13, no. 3, pp. 284-287, 1965.

[3] K. Kurihara and L. M. Beidler, "Taste-modifying protein from miracle fruit," Science, vol. 161, no. 3847, pp. 1241-1243, 1968.

[4] J. N. Brouwer, H. Van Der Wel, A. Francke, and G. J. Henning, "Miraculin, the sweetness-inducing protein from miracle fruit," Nature, vol. 220, no. 5165, pp. 373-374, 1968.

[5] K. Ito, T. Asakura, Y. Morita et al., "Microbial production of sensory-active miraculin," Biochemical and Biophysical Research Communications, vol. 360, no. 2, pp. 407-411, 2007.

[6] T. Hirai, A. M. Shohael, Y.-W. Kim, M. Yano, and H. Ezura, "Ubiquitin promoter-terminator cassette promotes genetically stable expression of the taste-modifying protein miraculin in transgenic lettuce," Plant Cell Reports, vol. 30, no. 12, pp. 22552265, 2011.

[7] K. Hiwasa-Tanase, T. Hirai, K. Kato, N. Duhita, and H. Ezura, "From miracle fruit to transgenic tomato: mass production of the taste-modifying protein miraculin in transgenic plants," Plant Cell Reports, vol. 31, no. 3, pp. 513-525, 2012.

[8] M. K. Wilken and B. A. Satiroff, "Pilot study of "miracle fruit" to improve food palatability for patients receiving chemotherapy," Clinical Journal of Oncology Nursing, vol. 16, no. 5, pp. E173E177, 2012.

[9] E. G. Achigan-Dako, D. A. Tchokponhoué, S. N’Danikou, J. Gebauer, and R. S. Vodouhè, "Current knowledge and breeding perspectives for the miracle plant Synsepalum dulcificum (Schum. et Thonn.) Daniell," Genetic Resources and Crop Evolution, vol. 62, no. 3, pp. 465-476, 2015.

[10] K. B. Swamy, S. A. B. Hadi, M. Sekaran, and M. R. A. Pichika, "The clinical effects of Synsepalum dulcificum: a review," Journal of medicinal food, vol. 17, no. 11, pp. 1165-1169, 2014.

[11] T. Misaka, "Molecular mechanisms of the action of miraculin, a taste-modifying protein," Seminars in Cell and Developmental Biology, vol. 24, no. 3, pp. 222-225, 2013.

[12] G. E. Inglett and D. Chen, "Contents of phenolics and flavonoids and antioxidant activities in skin, pulp, and seeds of miracle fruit," Journal of Food Science, vol. 76, no. 3, pp. C479-C482, 2011.

[13] Z. He, J. S. Tan, S. Abbasiliasi, O. M. Lai, Y. J. Tam, and A. B. Ariff, "Phytochemicals, nutritionals and antioxidant properties of miracle fruit Synsepalum dulcificum," Industrial Crops and Products, vol. 86, pp. 87-94, 2016.

[14] L. Q. Du, Y. X. Shen, X. M. Zhang, W. Prinyawiwatkul, and Z. M. Xu, "Antioxidant-rich phytochemicals in miracle berry (Synsepalum dulcificum) and antioxidant activity of its extracts," Food Chemistry, vol. 153, pp. 279-284, 2014.

[15] F. Cheng, S. Huang, M. Lin, and J. Lai, "Polyphenol measurement and antioxidant activity of miracle fruit," International Journal of Chemical Engineering and Applications, vol. 6, no. 3, pp. 211-214, 2015.

[16] H.-M. Wang, Y.-T. Chou, Z.-L. Hong et al., "Bioconstituents from stems of Synsepalum dulcificum Daniell (Sapotaceae) inhibit human melanoma proliferation, reduce mushroom tyrosinase activity and have antioxidant properties," Journal of the Taiwan Institute of Chemical Engineers, vol. 42, no. 2, pp. 204211, 2011.

[17] T.-Y. Chen, Z.-C. Kang, M.-T. Yen, M.-H. Huang, and B.-S. Wang, "Inhibitory effect of aqueous extracts from Miracle Fruit leaves on mutation and oxidative damage," Food Chemistry, vol. 169, pp. 411-416, 2015.

[18] Y.-C. Shi, K.-S. Lin, Y.-F. Jhai et al., "Miracle fruit (Synsepalum dulcificum) exhibits as a novel anti-hyperuricaemia agent," Molecules, vol. 21, no. 2, pp. 140-150, 2016.

[19] S. Kaki Bale, S. A. Abd Hadi, A. Latif, and A. Zubaidi, "Synsepalum dulcificum (Miracle fruit); a future potential antihyperglycemix herbal medicine," in Proceedings of the Conference in Natural Medicine, Canada, June 2012.

[20] C.-C. Chen, I.-M. Liu, and J.-T. Cheng, "Improvement of insulin resistance by miracle fruit (Synsepalum dulcificum) in fructoserich chow-fed rats," Phytotherapy Research, vol. 20, no. 11, pp. 987-992, 2006.

[21] H. Cao, "Polysaccharides from Chinese tea: recent advance on bioactivity and function," International Journal of Biological Macromolecules, vol. 62, pp. 76-79, 2013. 
[22] S.-P. Nie and M.-Y. Xie, "A review on the isolation and structure of tea polysaccharides and their bioactivities," Food Hydrocolloids, vol. 25, no. 2, pp. 144-149, 2011.

[23] A. K. Saha and C. F. Brewer, "Determination of the concentrations of oligosaccharides, complex type carbohydrates, and glycoproteins using the phenol-sulfuric acid method," Carbohydrate Research, vol. 254, pp. 157-167, 1994.

[24] C. C. Wang, S. C. Chang, B. S. Inbaraj, and B. H. Chen, "Isolation of carotenoids, flavonoids and polysaccharides from Lycium barbarum L. and evaluation of antioxidant activity," Food Chemistry, vol. 120, no. 1, pp. 184-192, 2010.

[25] F. W. Li, J. Gao, F. Xue, X. H. Yu, and T. Shao, "Extraction optimization, purification and physicochemical properties of polysaccharides from Gynura medica," Molecules, vol. 21, no. 4, pp. 397-409, 2016.

[26] J.-E. Li, S.-P. Nie, M.-Y. Xie, and C. Li, "Isolation and partial characterization of a neutral polysaccharide from Mosla chinensis Maxim. cv. Jiangxiangru and its antioxidant and immunomodulatory activities," Journal of Functional Foods, vol. 6, no. 1, pp. 410-418, 2014.

[27] P. Siddhuraju and K. Becker, "The antioxidant and free radical scavenging activities of processed cowpea (Vigna unguiculata (L.) Walp.) seed extracts," Food Chemistry, vol. 101, no. 1, pp. 1019, 2007.

[28] Y. L. Chew, Y. Y. Lim, M. Omar, and K. S. Khoo, "Antioxidant activity of three edible seaweeds from two areas in South East Asia," LWT-Food Science and Technology, vol. 41, no. 6, pp. 1067-1072, 2008.

[29] P. Xu, J. Wu, Y. Zhang, H. Chen, and Y. Wang, "Physicochemical characterization of puerh tea polysaccharides and their antioxidant and $\alpha$-glycosidase inhibition," Journal of Functional Foods, vol. 6, no. 1, pp. 545-554, 2014.

[30] Q. Yuan, Y. Xie, W. Wang et al., "Extraction optimization, characterization and antioxidant activity in vitro of polysaccharides from mulberry (Morus alba L.) leaves," Carbohydrate Polymers, vol. 128, pp. 52-62, 2015.

[31] H. Sies, "Strategies of antioxidant defense," European Journal of Biochemistry, vol. 215, no. 2, pp. 213-219, 1993.

[32] H. Chen, Z. Qu, L. Fu, P. Dong, and X. Zhang, "Physicochemical properties and antioxidant capacity of 3 polysaccharides from green tea, oolong tea, and black tea," Journal of Food Science, vol. 74, no. 6, pp. C469-C474, 2009.

[33] Y. Wang, X. Bian, J. Park, L. Ying, L. Qian, and P. Xu, "Physicochemical properties, in vitro antioxidant activities and inhibitory potential against $\alpha$-glucosidase of polysaccharides from ampelopsis grossedentata leaves and stems," Molecules, vol. 16, no. 9, pp. 7762-7772, 2011.

[34] Y. Wang, Z. Yang, and X. Wei, "Sugar compositions, $\alpha$-glucosidase inhibitory and amylase inhibitory activities of polysaccharides from leaves and flowers of Camellia sinensis obtained by different extraction methods," International Journal of Biological Macromolecules, vol. 47, no. 4, pp. 534-539, 2010.

[35] S. Zhang and X.-Z. Li, "Inhibition of $\alpha$-glucosidase by polysaccharides from the fruit hull of Camellia oleifera Abel," Carbohydrate Polymers, vol. 115, pp. 38-43, 2015.

[36] J. Cui, X. Gu, F. Wang, J. Ouyang, and J. Wang, "Purification and structural characterization of an $\alpha$-glucosidase inhibitory polysaccharide from apricot (Armeniaca sibirica L. Lam.) pulp," Carbohydrate Polymers, vol. 121, pp. 309-314, 2015. 

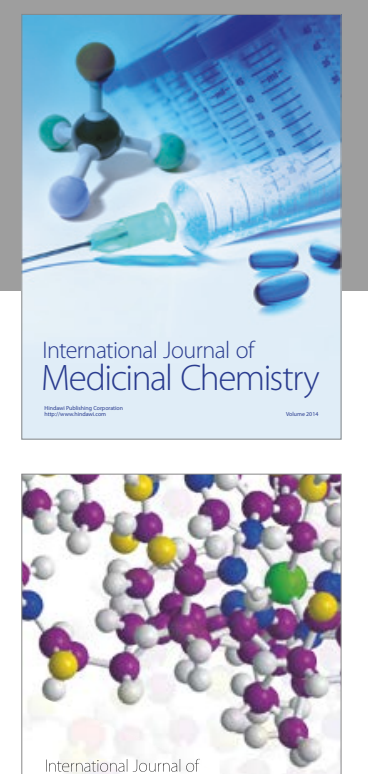

Carbohydrate Chemistry

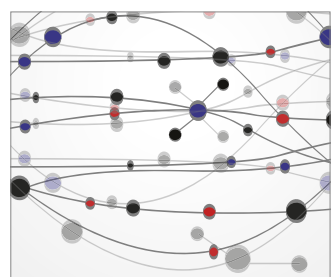

The Scientific World Journal
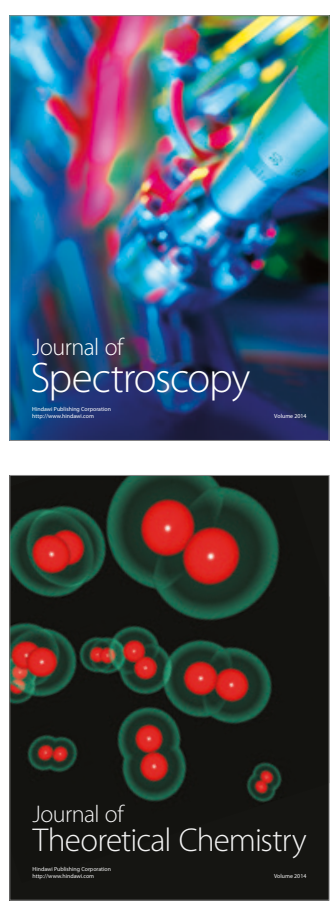
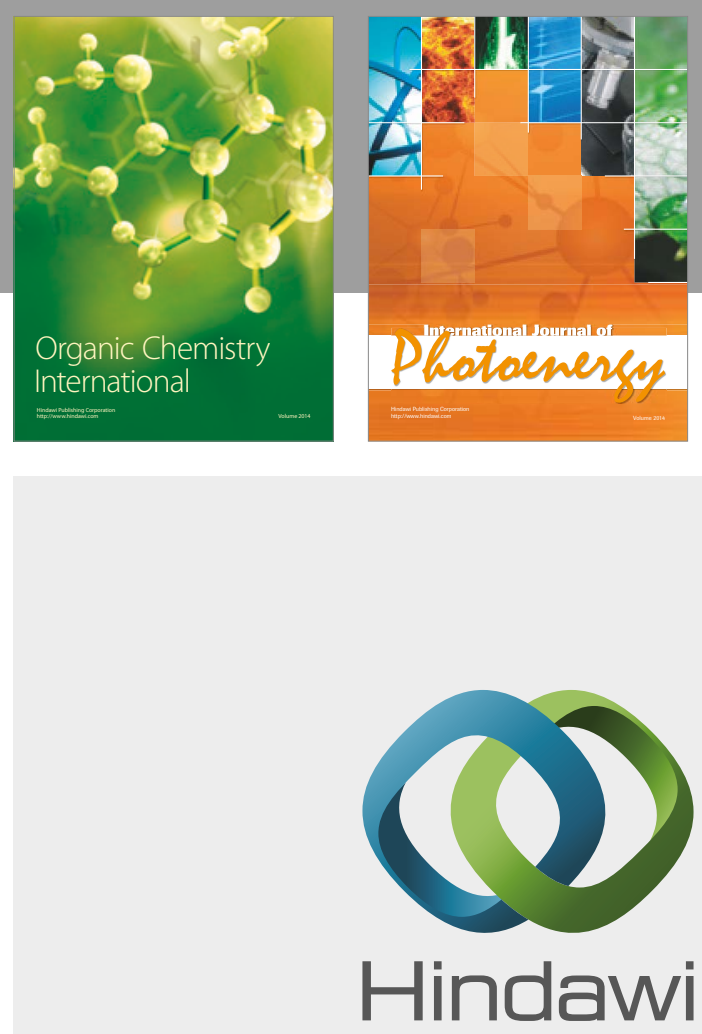

Submit your manuscripts at

https://www.hindawi.com

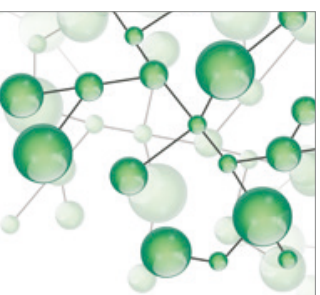

International Journal of

Inorganic Chemistry

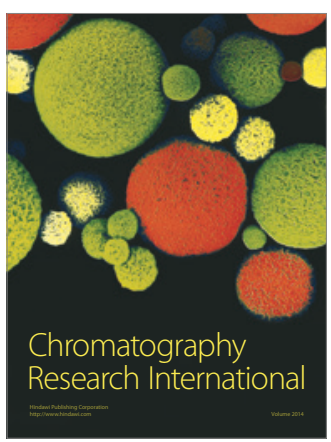

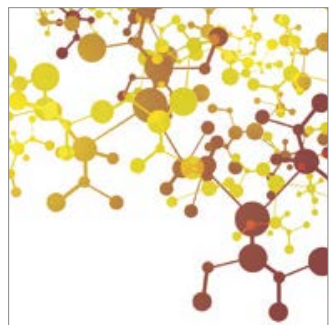

Applied Chemistry
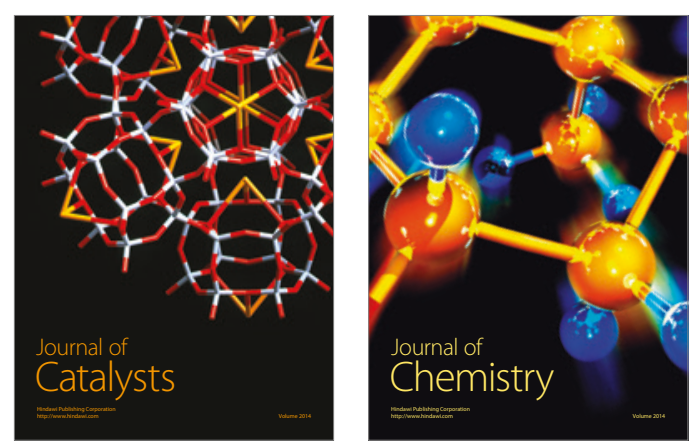
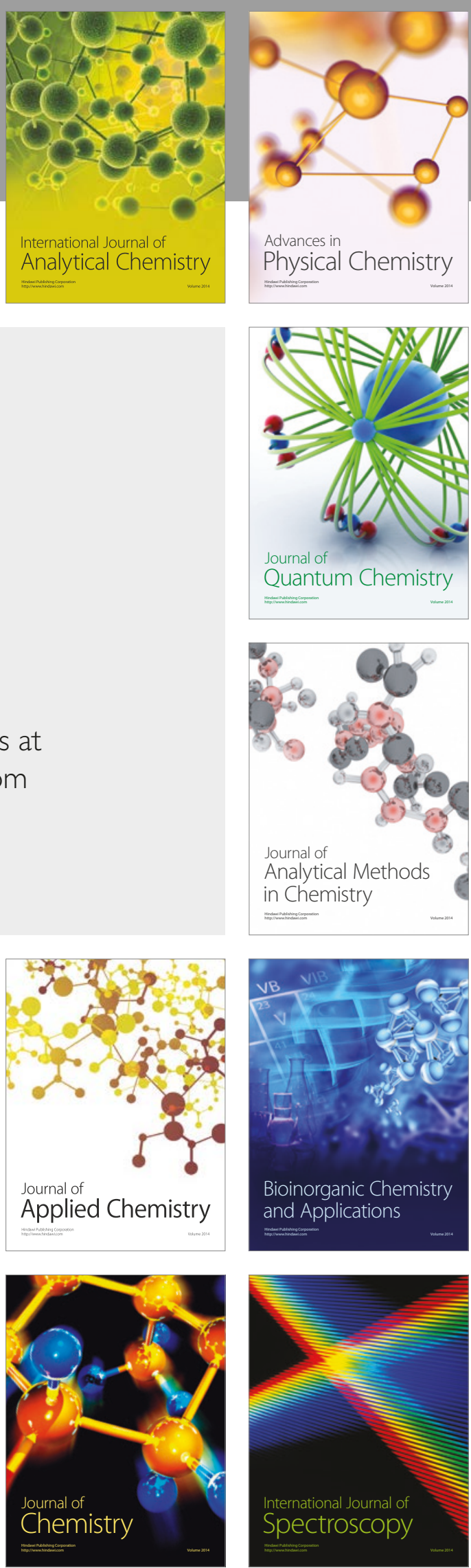